\title{
Modeling of an EMC Test-bench for Conducted Emissions in Solid State Applications
}

\author{
A.Micallef, C.Spiteri Staines and M.Apap \\ Department of Industrial Electrical Power Conversion \\ University of Malta \\ Malta
}

\begin{abstract}
In the area of EMC performance of electric motor drives, research is mainly focused on the EMC performance of inverter based drives. However, in most instances the soft starter is still an appropriate choice for motor control especially when accurate speed control of the load is not a specific requirement. The EMI generation of these converters has not been given the required importance, with only a few publications regarding this issue available in the last decades. A simulation model that considers the operation of solid state devices in an EMC conducted emission measurement laboratory environment was developed. The model was implemented in MATLAB ${ }^{\circledR}$ and Simulink ${ }^{\circledR}$ using a time domain approach. Models for the LISN, EMI receiver, power cables, thyristor power modules and induction motor load were developed and implemented. These models were developed so as to reflect the actual physics of the components and, where possible, model parameters were obtained through analysis of the geometry of the system through simplifications of the surrounding environment. The simulation was then used to gain insight on the EMI generation mechanisms of the solid state system.
\end{abstract}

\section{INTRODUCTION}

The market for soft starters is still growing steadily, thanks to their operational simplicity, relatively low cost and energy optimizing performance finding application in a wide variety of markets. EMI measurements are required to be made on a device after product completion to evaluate whether the emissions are within specified limits. Before any hardware prototype is completed, during the early stages of product development, modeling is the only solution to evaluate the EMI performance of the device and understand its EM behavior without the actual hardware by representing the main physical and electrical properties of the device. Simulation models to simulate and analyze the conducted EMI generation of solid state devices in a standard test environment were developed. The models developed can be used as a design tool to enable the determination of the required input filter according to the relevant standards.

For voltage output control, the thyristors require phase angle control which will cause high $\mathrm{dV} / \mathrm{dt}$ and $\mathrm{di} / \mathrm{dt}$ to occur which generate common mode currents and hence EMI. In solid state based devices, generation of common mode currents flowing to the ground through stray capacitances between the windings and the stator of electric machines, as well as between the phases and the ground conductor of energy cables is one of the major causes of these emissions. The models developed in this paper take into account parasitic capacitive coupling, skin and proximity effects in the power cables, parasitics of the thyristors and parasitics in the power module and also considers self capacitance, capacitive coupling to ground and mutual inductance in the motor load.

\section{A. Conducted Emission Testing}

Conducted emissions tests carried out at laboratory facilities require either a shielded environment or at least a suitably sized ground plane. Solid state devices are usually installed inside distribution boxes or similar earthed metal cases. Therefore, to ensure that the interaction between the DUT and ground plane is similar to that of the actual operating environment, the DUT must be floor standing and isolated from the ground plane. A block diagram of the test setup used for conducted emission measurements is shown in Fig. 1.

The system is required to measure the conducted emissions from a solid state device controlling an induction motor load. In this case, the solid state device (DUT), while operating in its normal conditions, must be operated in a configuration that maximizes the generated EMI. In addition to ensure that the measured noise is actually that generated by the equipment, the ambient noise present in the test location must be at a minimum of $6 \mathrm{~dB}$ from the limit being measured. The measurement equipment block consists either of scanning receivers, stepping receivers or spectrum analyzers and is used to measure the mains terminal disturbance voltage. The

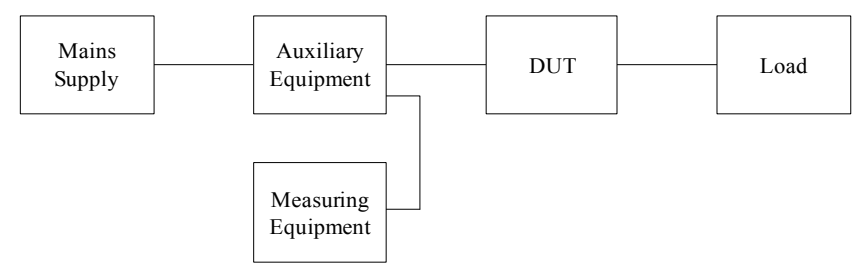

Fig. 1. Block diagram for the conducted emission measurement test setup 
auxiliary equipment mainly consist of artificial main networks (AMNs/ LISNs) to measure voltage disturbances on the mains terminals.

\section{TIME DOMAIN SimUlation MODELING}

This section describes the models that were used in the simulation which are interconnected as shown in Fig. 1 for the simulation of the complete system.

\section{A. AMN/LISN Model}

The LISN network model implemented was the $50 \Omega / /$ $50 \mu \mathrm{H}+5 \Omega \mathrm{V}$-network that enables coverage of both bands A and $\mathrm{B}$ with a single LISN (Band $\mathrm{A}$ is defined over the frequency band $9 \mathrm{kHz}$ to $150 \mathrm{kHz}$ while Band $\mathrm{B}$ is defined over $150 \mathrm{kHz}$ to $30 \mathrm{MHz})$. The schematic for the implementation of the V-network with the corresponding component values, are suggested in CISPR 16-1-2. The LISN monitors the noise on a single line and requires each line connecting the mains supply to the DUT to be interfaced with a LISN so as to enable monitoring of the conducted noise produced from the DUT on all lines.

Effects of the ground strap may affect the measurements by introducing a resonant frequency that distorts the impedance characteristics. Compensation for the additional impedance would be required for compliance in emissions. The schematic of the V-network was modified, as shown in Fig. 2, to include this ground strap effect by an LC resonant tank circuit between the ground plane and the reference plane of the LISN.

An analytical calculation of the coupling capacitance between the LISN and the ground plane is a complex procedure while the inductance varies according to the bonding strap length and type used. Experiments made in [1, 2] found that the typical LISN to ground plane capacitance was in the region of $500 \mathrm{pF}$. Therefore, so as to simplify calculations of compensation factors in the receiver, the resonant frequency should preferably be sufficiently over the $30 \mathrm{MHz}$ limit by selection of inductance $\mathrm{L} 3$. Values of $10 \mathrm{nH}$ to $40 \mathrm{nH}$ were suggested in $[1,2]$, for which the magnitude response was compliant to the standard, however the phase response did not comply. In this respect, a value of $2 \mathrm{nH}$ was

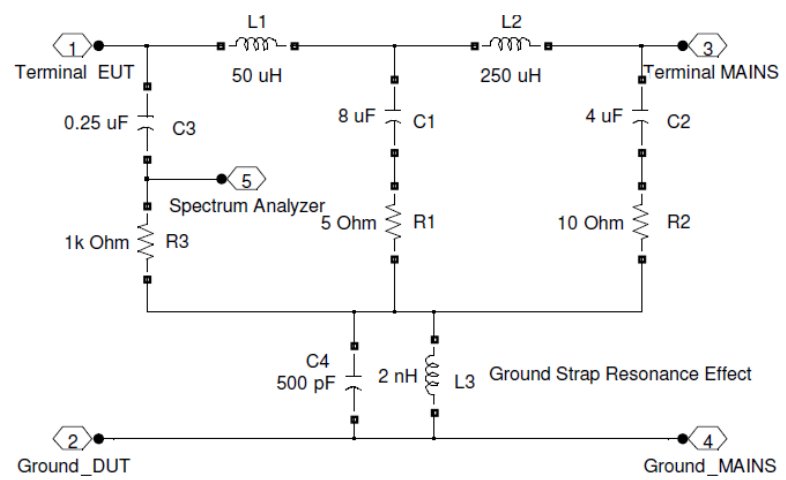

Fig. 2. Complete LISN Model

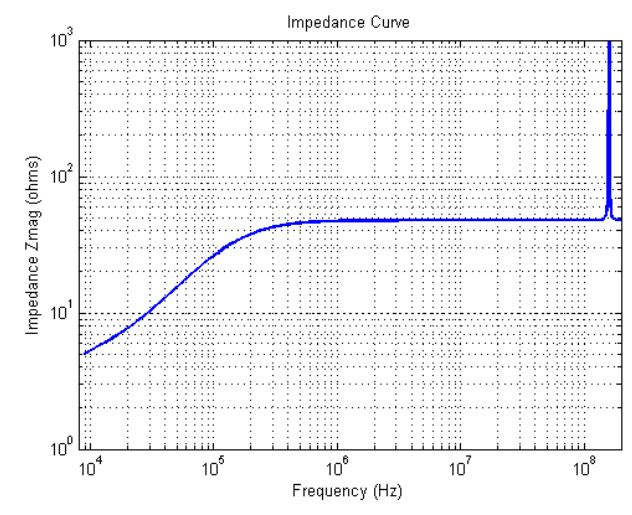

Fig. 3. LISN Model Impedance including effects of ground strap.

selected which achieved the impedance magnitude and phase plots as specified in the standard, with the addition of resonance at $159.22 \mathrm{MHz}$ due to the ground strap effects as shown in Fig. 3.

\section{B. Power Cables Model}

The cable system that was modeled is composed of three parallel $1.5 \mathrm{~mm}^{2}$ wires coated with PVC and laid uniformly over the ground plane as shown in Fig. 4. The DUT has no neutral and earth connections hence only the three phase cables were included in the model. Cable lengths of $1 \mathrm{~m}$ were specified by the standard from the LISN to the DUT and a similar length was used for cable from the DUT to the load.

The model used for the power cable was the lumped parameter transmission line model. However to increase the accuracy of the model, multiple T-sections (with constant parameters) were cascaded. The above model was further developed to include high frequency effects due to skin and proximity effects via ladder networks [3-6]. Parameters for the cable model were obtained by analytical solutions of the geometry of the system. A single transmission line segment, including high frequency effects, is shown in Fig. 5.

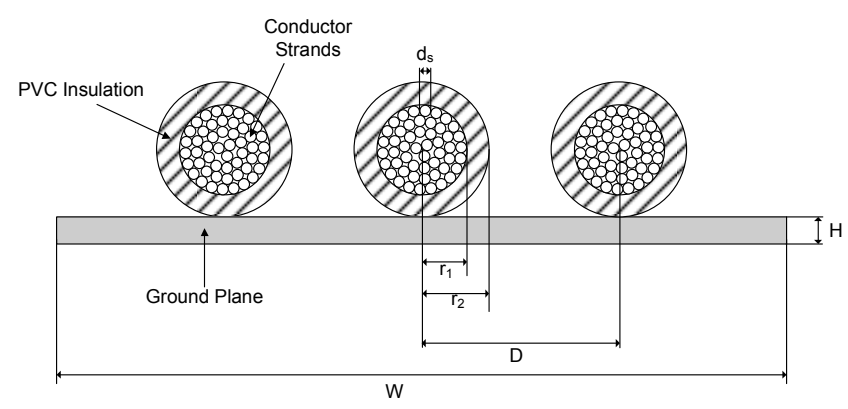

Fig. 4. Unshielded Cable Structure 


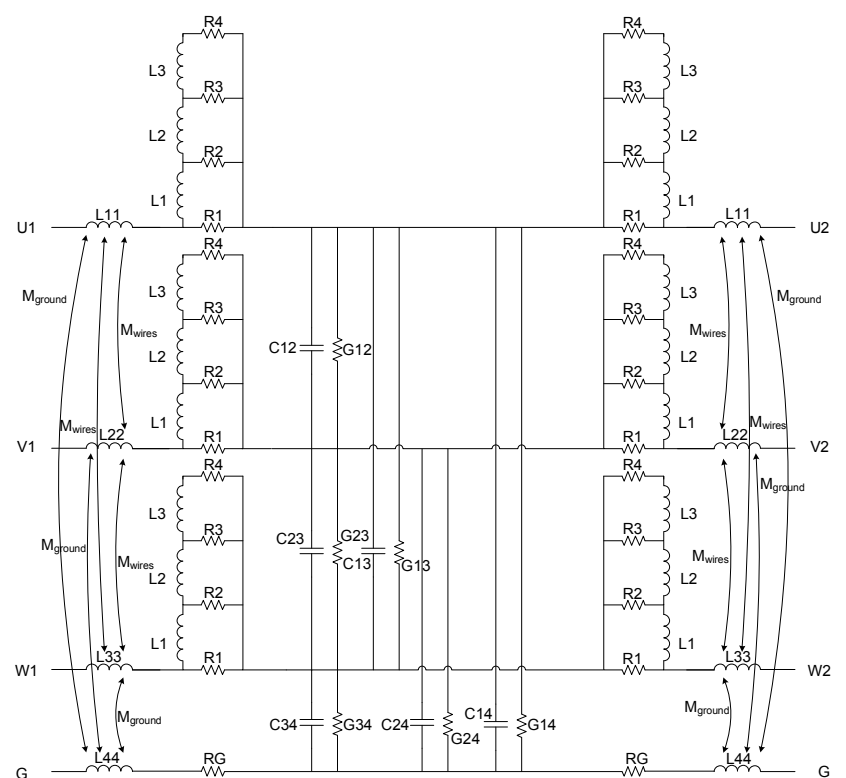

Fig. 5. Transmission Line Segment including High Frequency Effects

The minimum number of segments to be cascaded in the model was determined by maintaining the desired accuracy without increasing unnecessarily the simulation time. The required number of segments was determined by comparing the impedance response of the cables at different loads and for varying number of segments. The impedance response was measured between the ground plane and the shorted ends of the three phase wires. The number of segments which showed minimal change in the impedance compared to the next number of segments was chosen to be the required value. The
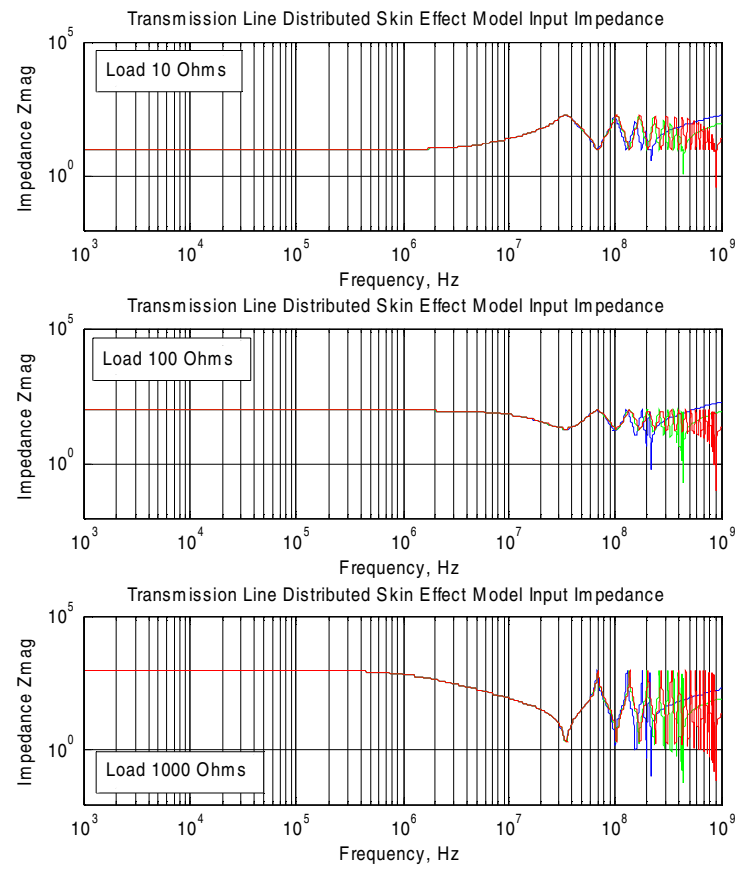

Fig. 6. Impedance Response for Different Segment Numbers resulting impedances for the simulated segments are shown in Fig. 6. For the parameters used in the simulation, increasing the number of segments beyond 10 segments exhibited no changes in impedance below $200 \mathrm{MHz}$. Hence 10 segments were used throughout the simulation.

\section{Induction Motor Model}

The induction motor model described in [7-9] was used in the simulations. Fig. 7 shows the schematic of the motor model. An analytical approach to obtain the parameters for the model is a complex procedure due to the nature of the windings and the various electromagnetic coupling effects involved inside the motor stator and case. It was not possible to carry out high frequency impedance measurements on the motor, thus model parameters obtained from [9] were used in the simulation to verify the overall model.

To verify the implemented model against the model described in [9], simulations of the line-to-line and line-to-ground impedances over the required frequency range were performed and the corresponding impedance responses are shown in Fig. 8 and Fig. 9. The curves obtained were equivalent to those obtained in [9] thus confirming the operation of the implemented model.

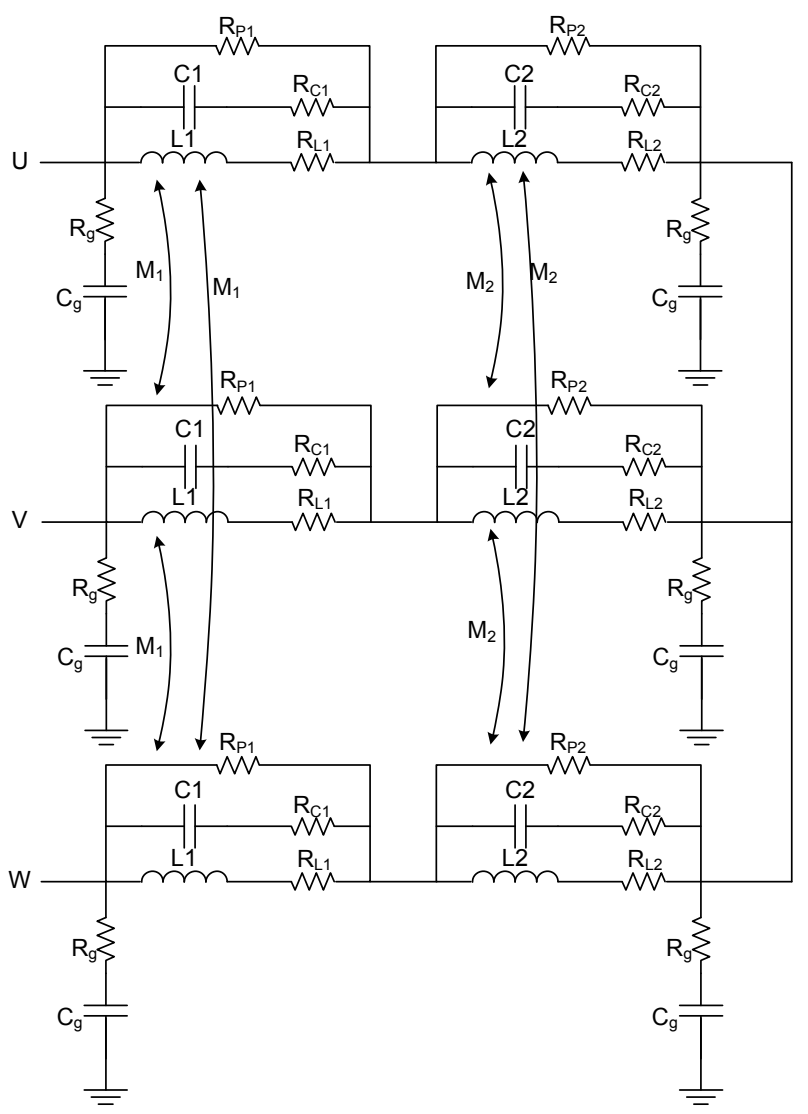

Fig. 7. Induction Motor High Frequency Model 


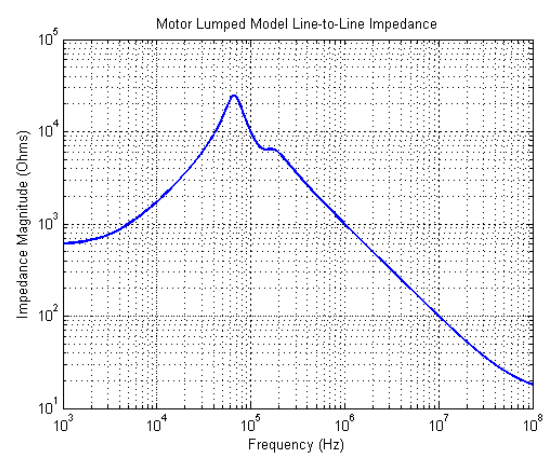

Fig. 8. Induction Motor Line to Line Impedance Magnitude.

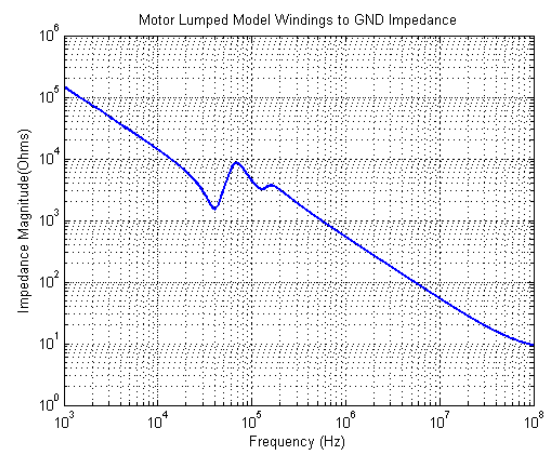

Fig. 9. Induction Motor Line to Ground Impedance Magnitude.

\section{Thyristors Model}

Due to the wide range of characteristics modeled and its simplicity, the two transistor - three resistor (2T-3R) thyristor model $[10,11]$ was implemented to model the operation of the IXYS CWP25 thyristor chip [12].The model is based on the two transistor model derived from the physical structure of the thyristor. Bipolar transistor models were based on the EbersMoll model. A piecewise linear approximation was used for the diode models, as algebraic loops occurred in the exponential model which increased the simulation times and caused convergence errors for particular conditions.

The 2T-3R thyristor model was then used to model the static I-V characteristics of the thyristors. Curve fitting was used in this case to obtain the model parameters. A comparison between the forward conducting characteristics of the simulation model and physical measurements is shown in Fig 10 . The model was seen to follow faithfully the measured results for currents less than 55A, while for larger currents the error increases with the current magnitude.

For EMI simulations, the model must also take into account the parasitics due to the physical structure of the thyristor by adding the second order effects in the transistor models. Fig. 11 shows the 2T-3R thyristor model by combination of the two high frequency transistor models. The values of the thyristor parasitics can be obtained either by knowledge of the manufacturing data (areas, doping levels, etc..) or by curve fitting of the switching characteristics.

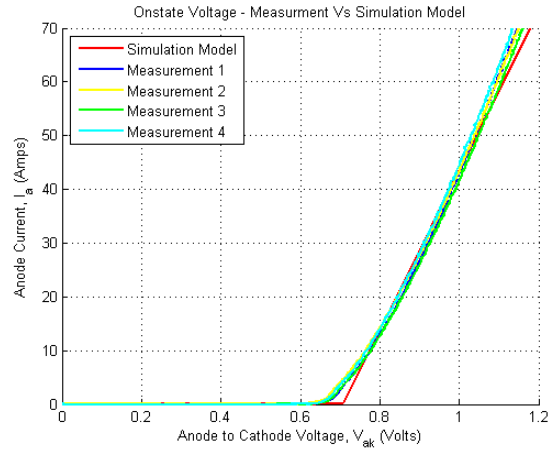

Fig. 10. Forward Conducting Characteristics.

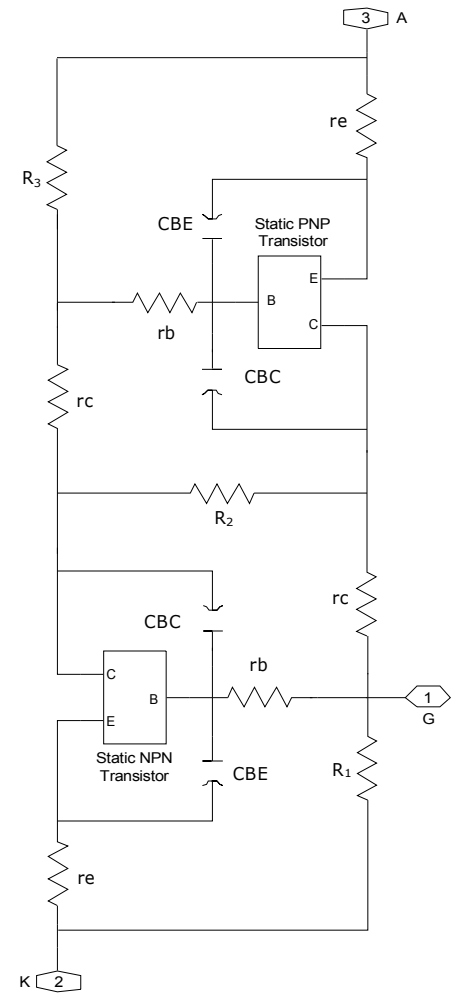

Fig. 11. 2T-3R High Frequency Equivalent.

\section{E. Power Module Model}

Thyristor dies such as the IXYS CWP25, are usually assembled into a module, typically with the two anti-parallel dies in a single module. The power module configuration considered is shown in Fig. 12 and Fig. 13. The complete high frequency electrical modeling of the power module is shown in Fig. 14. The two power terminals, gate terminals and interconnects were each modeled using the short transmission line model and hence include only the respective series R-L components. The DCB was modeled as two $\pi$-section transmission lines, having a common return path through the baseplate that was assumed to be coupled to the ground plane. An additional resistance (ESR) was added in series to the capacitance to model the losses that occur in this parasitic capacitive coupling. Mutual coupling (inductive, MDCB) was also assumed between the two DCB copper tracks. The values 
of the parameters were based on low frequency calculations and high frequency effects such as skin and other proximity effects were ignored. The electrical representation of the parasitics in the power module was obtained from analysis of the geometry of the system and material properties.

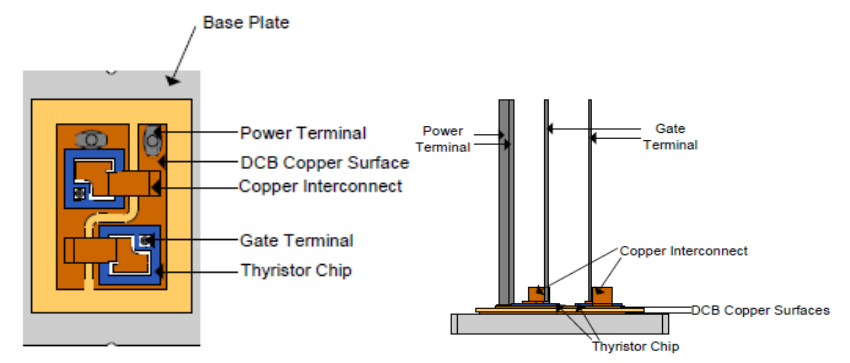

Fig. 12. Plan View of Power Module.

Fig. 13. Side View of Power Module.

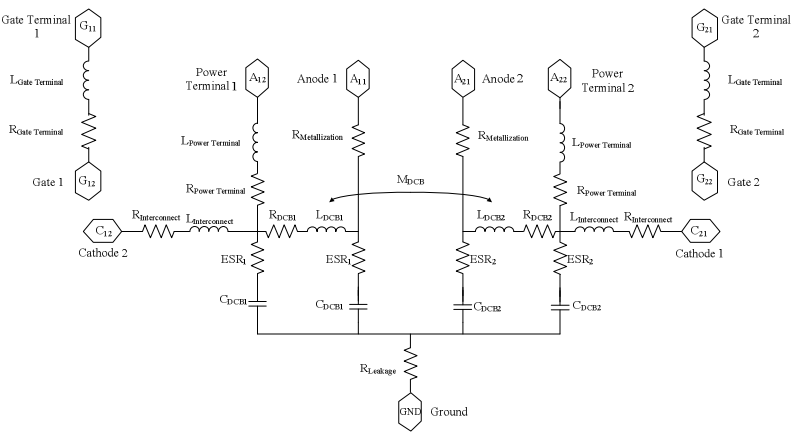

Fig. 14. Parasitics of the power module

\section{F. EMI Receiver Model}

\section{1) CISPR Receiver Model}

For accurate measurements and suitable comparison to the limits provided in the required standards, a receiver model compliant to CISPR 16-1-1 was developed. The LISN noise voltage output was processed separately from the system model to obtain the emission measurements so as to decrease simulation times. Peak detection was implemented since the simulation times for the quasi-peak were expected to last around a month as opposed to the peak method which required a computation time of a few days. The frequency step used was dependent on the frequency range being measured and was selected to be equal to half the resolution bandwidth of the receiver. This implies that for band A, the frequency step was $100 \mathrm{~Hz}$ while for band $\mathrm{B}$ a frequency step of $4.5 \mathrm{kHz}$ was used to obtain coverage of the whole spectrum. The implementation for the receiver model is shown in figure 15 where the LISN data is loaded in the lookup table at start up and the measured frequency is swept by the receiver control block. Separate measurement receivers for both bands were implemented, due to their different requirements. Both receivers processed data in parallel and the receiver control block selects the receiver according to the frequency range being scanned.
During tests carried out on the receiver model, emission signals were seen to be non-stationary transient types. A dwell time of $20 \mathrm{~ms}$ was used during the simulations which results in $160.92 \mathrm{~s}$ of measurements to be simulated. Due to the $400 \mathrm{MHz}$ sampling frequency, the simulation time for a single scan was seen to take several days.

\section{2) STFT-based Receiver Model}

Due to the intensive processing required and the extremely long simulation times of the CISPR implementation, an alternative method was developed to obtain conducted emission plots within shorter simulation times. The most common method for analyzing the frequency spectrum of discrete time data is through the use of the discrete Fourier transform (DFT). Since non-stationary signals have a time varying spectrum, calculation of the DFT for the whole signal becomes inadequate to obtain an actual indication of the spectrum. To achieve a combined time domain - frequency domain analysis, an algorithm based on the STFT was developed to process the noise voltages from the LISN and obtain the frequency plot of the emission levels.

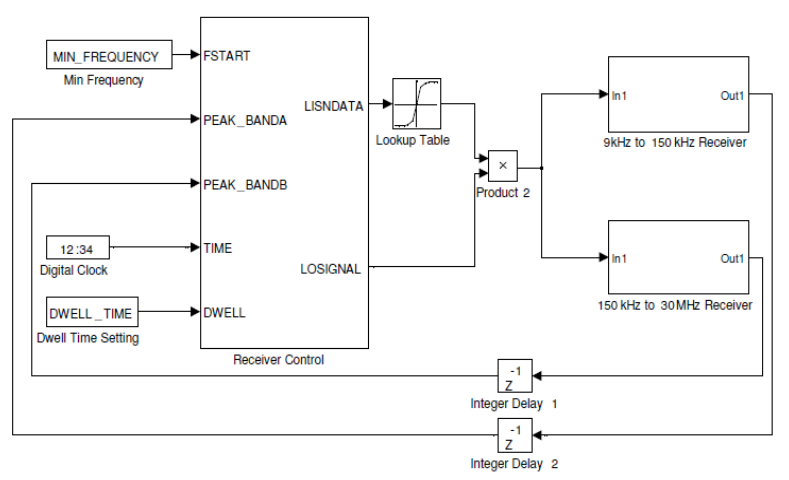

Fig. 15. CISPR Receiver Model

The principle of operation of the implemented algorithm is given in Fig. 16 and Fig. 17. The input data block from the LISN is scanned by a sliding rectangular window of a predefined sample size (L) during which the signal was assumed to be stationary. On each windowed data block, an $\mathrm{N}$-point DFT was then performed to obtain the spectrum of each window. The resolution of the DFT should be equal to that of the EMI receiver to enable a correct comparison and is dependent on the frequency band being analyzed.

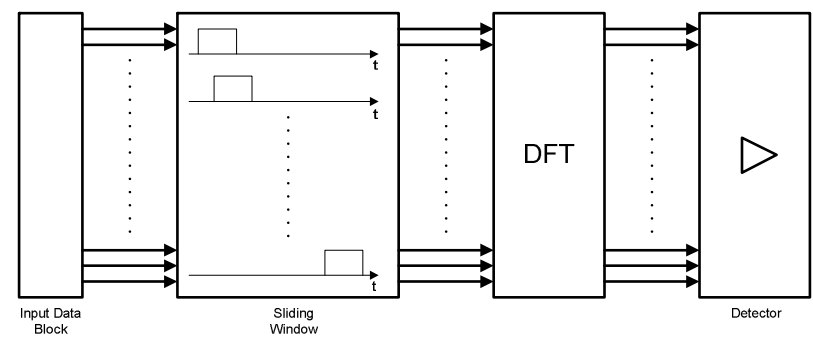

Fig. 16. General block diagram of STFT data processing algorithm 


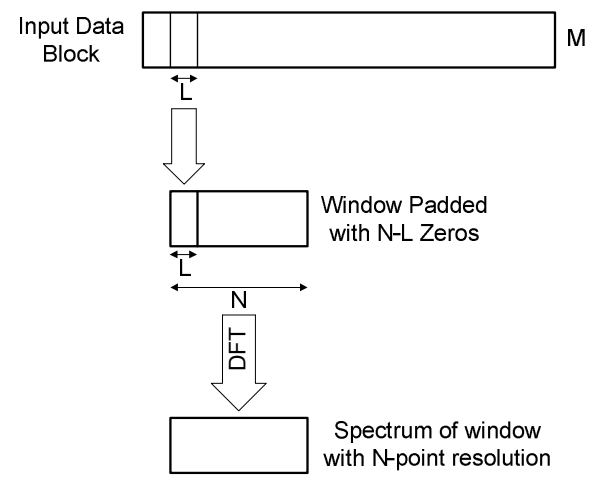

Fig. 17. Data processing block diagram of STFT algorithm

The performance of the STFT algorithm over band B, was verified by comparing the emission plots obtained using a single frequency of $1 \mathrm{MHz}$ and for three overlapping frequencies namely $1 \mathrm{MHz}, 10 \mathrm{MHz}$ and $20 \mathrm{MHz}$. The emission waveforms obtained, shown in Fig. 18 and Fig. 19 respectively, showed a maximum error of $-0.4 \mathrm{~dB}$ between the two implementations. Similarly the performance of the STFT algorithm over band A was verified by comparing the emission plots obtained for a single frequency of $100 \mathrm{kHz}$ and for three overlapping frequencies namely $10 \mathrm{kHz}, 50 \mathrm{kHz}$ and $100 \mathrm{kHz}$. The emission waveforms obtained, shown in Fig. 20 and Fig. 21 respectively, show a maximum error of $-0.2 \mathrm{~dB}$ between the two implementations.

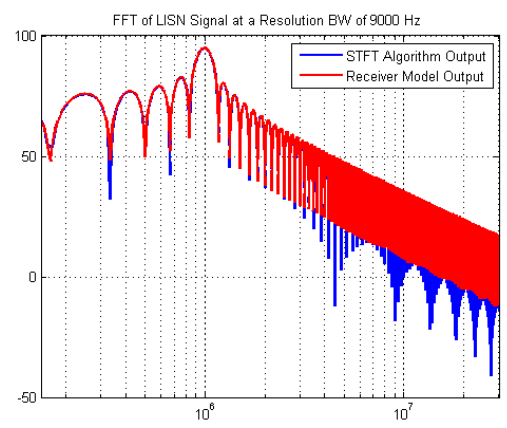

Fig. 18. Band B Response to $1 \mathrm{MHz}$ transient.

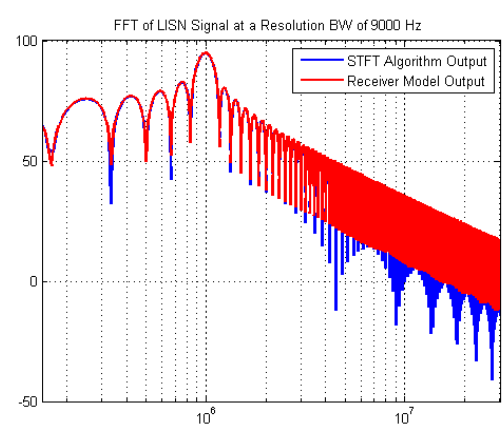

Fig. 19. Band B Response to a transient with frequencies $1 \mathrm{MHz}$, $10 \mathrm{MHz}$ and $20 \mathrm{MHz}$.

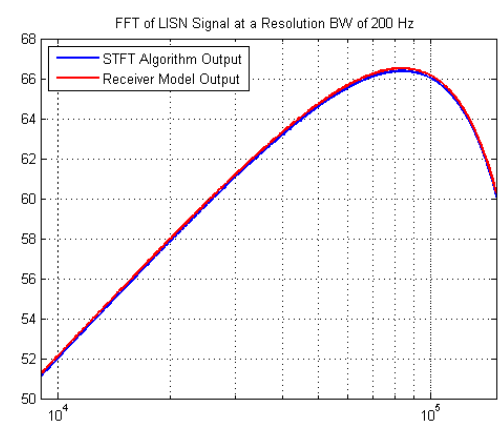

Fig. 20. Band A Response to $100 \mathrm{kHz}$ transient.

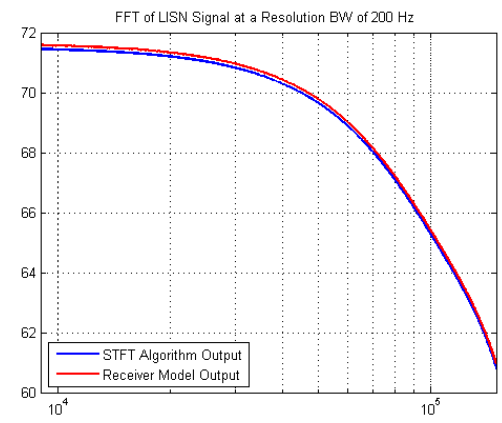

Fig. 21. Band A Response to a transient with frequencies $10 \mathrm{kHz}, 50 \mathrm{kHz}$ and $100 \mathrm{kHz}$.

The results showed that, the STFT algorithm achieved similar results to those achieved by the CISPR implementation with peak detection. However, the execution time of the STFT algorithm was much faster than the CISPR implementation with simulation times ranging from a few seconds to not more than 6 hours. The execution speed of the STFT algorithm depends on the window size, the amount of overlap and the size of the N-FFT that is carried out for each block. Hence the STFT algorithm can be considered as a viable alternative to the CISPR implementation.

TABLE I. STFT ALGORITHM PARAMETERS

\begin{tabular}{|c|c|c|}
\hline Symbol & Quantity & Value in number of samples \\
\hline M & Input Data Length & 8000000 \\
\hline L & Rectangular Window Size & 7200 \\
\hline N & DFT Resolution 9kHz (Band B) & 44444 \\
\hline N & DFT Resolution 200Hz (Band A) & 2000000 \\
\hline
\end{tabular}

\section{RESULTS}

The complete system (Fig. 1) was modeled using the modules described in sections A to F, with the aim to analyze the effect of variations in the firing angle on the emissions. The firing angle has a direct effect on the measured emission levels as shown in Fig. 22, which illustrates the emission levels for firing angles greater than 60 degrees. As expected, the maximum emissions were observed at a firing angle of 90 degrees where the maximum $\mathrm{dV} / \mathrm{dt}$ occurs. The emission 
spectrum envelope for band B was seen to remain fairly uniform as the firing angle was changed, except for changes in the amplitude.

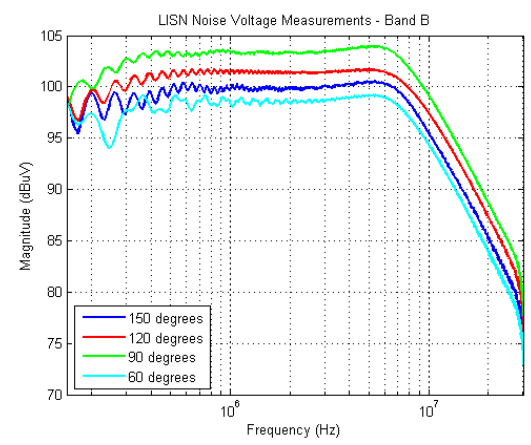

Fig. 22. Emissions for different firing angles. Band B Measurements.

\section{CONCLUSION}

Models for the test setup used in the measurement of conducted emissions were developed. Parameters for the models, except the induction motor, were obtained by an analytical solution. Parameters for the motor model were obtained from [9] and the impedance response of the motor was seen to be equivalent to that in [9] hence confirming the implementation. An STFT algorithm was developed to obtain the conducted emissions from the LISN voltages. The algorithm has shown a maximum error of $0.4 \mathrm{~dB}$ while the computation speed was reduced significantly when compared to the CISPR implementation. From simulations carried out that consider the effect of the firing angle on the emissions, emissions were observed to be a maximum at peak switching.
Emission analysis is currently being carried out to analyze the main noise contributors of the modeled system.

\section{REFERENCES}

[1] Calibration and use of artificial mains networks and absorbing clamps [Online]. Available: http://www.elmac.co.uk

[2] T. Williams and G.R. Orford, "Best practice use of the CISPR AMN/LISN", International Conference and Exhibition on Electromagnetic Compatibility, York, 1999.

[3] S. Kim and D.P. Neikirk, "Compact Equivalent Circuit Model for the Skin Effect", IEEE MTT-S International Microwave Symposium Digest, Vol. 3, San Francisco, 1996.

[4] S. Mei and Y.I. Ismail, "Modeling Skin and Proximity Effects With Reduced Realizable RL Circuits", IEEE Trans. Very Large Scale Integr. (VLSI) Syst., Vol. 12, No. 4, April 2004

[5] B. Mukherjee, L. Wang and A. Pacelli, "A Practical Approach to Modeling Skin Effect in On-Chip Interconnects", Proc. ACM Great Lakes symposium on VLSI, Boston, 2004, pp. 266-270

[6] S. Kim and D.P. Neikirk, "Compact Equivalent Circuit Model for the Skin Effect", IEEE MTT-S International Microwave Symposium Digest, Vol. 3, San Francisco, 1996.

[7] G. Grandi, D. Casadei and U. Reggiani, "Common- and DifferentialMode HF Current Components in AC Motors Supplied by Voltage Source Inverters", IEEE Trans. Power Electr., Vol. 19, No. 1, January 2004.

[8] G. Grandi, D. Casadei and U. Reggiani, "Equivalent Circuit of Mush Wound AC Windings for High Frequency Analysis", IEEE International Symposium on Industrial Electronics, Guimaraes, July 1997.

[9] G. Grandi, D. Casadei and A. Massarini, "High Frequency Lumped Parameter Model for AC Motor Windings", European Conference on Power Electronics and Applications, Trondheim, September 1997.

[10] M.H. Rashid, Power Electronics Handbook, Academic press, 2001.

[11] C.L. Tsay et al., "A High Power Circuit Model For The Gate Turn-off Thyristor", IEEE Power Electronics Specialists Conference, June 1990, pp. 390-397.

[12] CWP 25 Datasheet [Online]. Available: http://www.ixys.com 